\title{
On integers of the form $p+2^{k}$
}

\author{
by \\ Laurent Habsieger and Xavier-François Roblot (Lyon)
}

1. Introduction. Thoughout this paper, the symbol $p$ will denote a prime and $k$ will be a nonnegative integer. Romanov [5] proved that the integers of the form $p+2^{k}$ have positive density. He also raised the following question: does there exist an arithmetic progression consisting only of odd numbers, no term of which is of the form $p+2^{k}$ ? Erdös [1] found such an arithmetic progression by considering integers which are congruent to 172677 modulo $5592405=\left(2^{24}-1\right) / 3$. Thus the density of numbers of the form $p+2^{k}$ is less than $1 / 2$, the trivial bound obtained from the odd integers. For convenience we introduce

$$
\underline{d}=\liminf _{x \rightarrow \infty} \frac{\#\left\{p+2^{k} \leq x\right\}}{x / 2} \quad \text { and } \quad \bar{d}=\limsup _{x \rightarrow \infty} \frac{\#\left\{p+2^{k} \leq x\right\}}{x / 2} .
$$

The aim of this paper is to give an explicit version of the estimates $0<\underline{d} \leq$ $\bar{d}<1$.

THEOREM 1. We have

$$
0.1866<\underline{d} \leq \bar{d}<0.9819 .
$$

This range is pretty large and Bombieri conjectured the more precise upper bound 0.868 (see [4]).

In Section 2, we obtain the lower bound $0.1866<\underline{d}$, by slightly refining a straightforward application of a recent result of Pintz and Ruzsa [3], in their study of Linnik's approximation of the Goldbach problem (see also [2]). In Section 3 , we get the upper bound, using computations on residue classes.

2. The lower bound. Let $N$ be a large integer and put $L=\lfloor\log N / \log 2\rfloor$. Define the functions

$$
r(n)=\#\left\{(p, k): n=p+2^{k}, p \leq N, 1 \leq k \leq L\right\}
$$

2000 Mathematics Subject Classification: Primary 11P32; Secondary 11Y35, 11Y60.

Partially supported by the European Community IHRP Program, within the Research Training Network "Algebraic Combinatorics in Europe", grant HPRN-CT-2001-00272. 
and

$s(N)=\#\left\{\left(p_{1}, p_{2}, k_{1}, k_{2}\right): p_{1}-p_{2}=2^{k_{2}}-2^{k_{1}}, p_{j} \leq N, 1 \leq k_{j} \leq L, j=1,2\right\}$

so that

$$
s(N)=\sum_{n=1}^{N} r^{2}(n) .
$$

Pintz and Ruzsa [3] proved the following lemma.

Lemma 1. For $N$ large enough, we have

$$
s(N) \leq \frac{2}{\log ^{2} 2} C N
$$

where $C<5.3636$.

Let $d(N)$ denote the number of positive integers $n \leq N$ which may be written in the form $n=p+2^{k}$. The Cauchy-Schwarz inequality implies easily that

$$
(\pi(N) L)^{2} \leq d(N) s(N)
$$

where $\pi(N)$ denotes the number of primes $p \leq N$. We deduce from Lemma 1 and from the prime number theorem that $2 C d(N) \geq(1+o(1)) N$, and the lower bound $\underline{d} \geq 1 / C>0.1864$ follows from the definitions.

To get the bound from the theorem, we need further notations. Put

$$
\varepsilon_{N}=\frac{\sum_{1 \leq n \leq N, r(n)>0} r(n)}{\sum_{1 \leq n \leq N, r(n)>0} 1} \quad \text { and } \quad \varepsilon=\frac{2}{\underline{d} \log 2} .
$$

By the definitions, there exists a subsequence of $\left(\varepsilon_{N}\right)_{N \in \mathbb{N}}$ which converges to $\varepsilon$. Let us now refine the Cauchy-Schwarz inequality by studying

$$
\Delta_{N}=\sum_{1 \leq n \leq N, r(n)>0}\left(r(n)-\varepsilon_{N}\right)^{2},
$$

so that

$$
\begin{aligned}
\Delta_{N} & =\sum_{1 \leq n \leq N} r^{2}(n)-\frac{\left(\sum_{1 \leq n \leq N} r(n)\right)^{2}}{\sum_{1 \leq n \leq N, r(n)>0} 1}=s(N)-\frac{(\pi(N) L)^{2}}{d(N)} \\
& \leq\left(5.3636-\frac{1}{\underline{d}}+o(1)\right) \frac{2 N}{\log ^{2} 2}
\end{aligned}
$$

for infinitely many $N$. Without loss of generality we may assume that $\varepsilon \in$ ]15, 15.5 [: otherwise we would get either $\underline{d} \geq 0.19$, which would be better, or 
$\underline{d} \leq 0.1862$, which is false. For infinitely many $N$ we thus have

$$
\begin{aligned}
\Delta_{N} \geq \sum_{1 \leq n \leq N, r(n)>0}\left(15-\varepsilon_{N}\right)^{2} & \geq\left(\sum_{1 \leq n \leq N, r(n)>0}(15-\varepsilon)^{2}+o(1)\right) N \\
& =\left(\frac{\underline{d}}{2}\left(15-\frac{2}{\underline{d} \log 2}\right)^{2}+o(1)\right) N .
\end{aligned}
$$

We deduce from these estimates the inequality

$$
\frac{\underline{d}}{2}\left(15-\frac{2}{\underline{d} \log 2}\right)^{2} \leq \frac{2}{\log ^{2} 2}\left(5.3636-\frac{1}{\underline{d}}\right),
$$

which may be written as

$$
56.25 \log ^{2} 2 \underline{d}^{2}-(15 \log 2+5.3636) \underline{d}+1 \leq 0 .
$$

The lower bound $\underline{d} \geq 0.1866$ then follows.

\section{The upper bound}

A. Basic ideas. Let us introduce further notations. Let $M$ be a positive odd integer and let $\omega$ denote the order of 2 in $(\mathbb{Z} / M \mathbb{Z})^{*}$. For $\bar{m}$ a residue class modulo $M$, put

$$
f_{M}(\bar{m})=\left\{\bar{k} \in \mathbb{Z} / \omega \mathbb{Z}: \bar{m}-2^{\bar{k}} \in(\mathbb{Z} / M \mathbb{Z})^{*}\right\}
$$

and

$$
\delta_{M}(\nu)=\left|\left\{\bar{m} \in \mathbb{Z} / M \mathbb{Z}:\left|f_{M}(\bar{m})\right|=\nu\right\}\right| .
$$

The basic tool to get an upper bound for $\bar{d}$ is the following lemma.

LEMMA 2. With the previous notations, we have

$$
\bar{d} \leq \sum_{\nu=0}^{\omega} \delta_{M}(\nu) \min \left(\frac{1}{M}, \frac{2 \nu}{\omega \varphi(M) \log 2}\right),
$$

where $\varphi$ denotes Euler's function.

Proof. Let $\bar{m}$ be a congruence class modulo $M$, with $\left|f_{M}(\bar{m})\right|=\nu$. Let us study the proportion of odd integers congruent to $\bar{m}$ that may be written in the form $p+2^{k}$. This proportion is clearly at most $1 / M$, and we only need to prove the alternative upper bound.

Since all the primes but a finite number are invertible modulo $M$, there exist $\nu$ congruence equations $\bar{m}=\bar{p}_{i}+2^{\bar{k}_{i}}, i \in\{1, \ldots, \nu\}$, such that all but finitely many representations $p+2^{k}$ come from one of these congruence equations. The number of primes up to $N$ which are congruent to $p_{i}$ modulo $M$ is asymptotic to $N /(\varphi(M) \log N)$, while the number of powers of 2 which are congruent to $2^{k_{i}}$ modulo $M$ is asymptotic to $\log N /(\omega \log 2)$. Thus the number of integers congruent to $\bar{m}$ that may be written in the form $p+2^{k}$ is at most $(\nu /(\varphi(M) \omega \log 2)+o(1)) N$. This implies that the proportion of 
odd integers enjoying these properties is at most $2 \nu /(\varphi(M) \omega \log 2)$ and the lemma follows.

This lemma provides a nontrivial upper bound for $\bar{d}$ as soon as there exist residue classes $\bar{m}$ modulo $M$ such that

$$
f_{M}(\bar{m})<\frac{\omega \varphi(M) \log 2}{2 M},
$$

a condition that occurs for a small number of classes. The main problem is to compute the distribution of the $f_{M}(\bar{m})$ 's in an efficient way. The direct computation of all the $f_{M}(\bar{m})$ 's is quickly limited by memory problems. However one can obtain significant results this way.

Take $M=23205=\left(2^{24}-1\right) / 723$, so that $\omega=24$ and $\varphi(M)=9216$. Condition (1) is equivalent to $f_{M}(\bar{m}) \leq 3$. We find

$$
\left(\delta_{M}(0), \delta_{M}(1), \delta_{M}(2), \delta_{M}(3)\right)=(0,48,720,320),
$$

and we get this way $\bar{d} \leq 0.985049$.

B. Refined algorithms and results. It appears that the function $f_{M}$ takes very few possible values, when compared to the set of subsets of $\mathbb{Z} / \omega \mathbb{Z}$. So let us introduce

$$
g_{M}(I)=\left\{\bar{m} \in \mathbb{Z} / M \mathbb{Z}: f_{M}(\bar{m})=I\right\} \quad \text { and } \quad G_{M}(I)=\left|g_{M}(I)\right|
$$

for $I \subset \mathbb{Z} / \omega \mathbb{Z}$. Note that

$$
\delta_{M}(\nu)=\sum_{|I|=\nu} G_{M}(I)
$$

So it is sufficient to know the distribution of the $G_{M}(I)$ 's to compute an upper bound for $\bar{d}$.

The main advantage of the function $g_{M}$ is that it is easily computable by induction on the number of prime factors of $M$. The initial case is given by $g_{0}(\{0\})=\{0\}$.

Let $M_{1}, M_{2}$ be two positive odd squarefree integers, with $M_{2}=p M_{1}$ for some prime $p$ not dividing $M_{1}$. Let $\omega_{1}, \omega_{2}$ and $\omega_{p}$ denote the order of 2 in $\left(\mathbb{Z} / M_{1} \mathbb{Z}\right)^{*},\left(\mathbb{Z} / M_{2} \mathbb{Z}\right)^{*}$ and $(\mathbb{Z} / p \mathbb{Z})^{*}$, respectively. The image of $f_{p}$ is easy to compute. There is the subset

$$
I_{p, 0}=\left\{\overline{2}^{\bar{k}} \in(\mathbb{Z} / p \mathbb{Z})^{*}: \bar{k} \in \mathbb{Z} / \omega_{p} \mathbb{Z}\right\}
$$

with $G_{p}\left(I_{p, 0}\right)=p-\omega_{p}$, and for each $\bar{j} \in \mathbb{Z} / \omega_{p} \mathbb{Z}$ the subset

$$
I_{p, \bar{j}}=\left\{\overline{2}^{\bar{k}} \in(\mathbb{Z} / p \mathbb{Z})^{*}: \bar{k} \in \mathbb{Z} / \omega_{p} \mathbb{Z}, \bar{k} \neq \bar{j}\right\}
$$

with $G_{p}\left(I_{p, \bar{j}}\right)=1$. Now, let $I_{2}$ and $I_{p}$ be in the image of $f_{M_{2}}$ and $f_{p}$ respectively. Denote by $\widetilde{I}_{2}$ and $\widetilde{I_{p}}$ the subsets of $\mathbb{Z} / M_{1} \mathbb{Z}$ which are inverse images of $I_{2}$ and $I_{p}$ under the map on subsets induced by the natural surjections 
$\mathbb{Z} / M_{1} \mathbb{Z} \rightarrow \mathbb{Z} / M_{2} \mathbb{Z}$ and $\mathbb{Z} / M_{1} \mathbb{Z} \rightarrow \mathbb{Z} / p \mathbb{Z}$ respectively. Then it is easy to see that $\widetilde{I}_{2} \cap \widetilde{I}_{p}$ is in the image of $f_{M_{1}}$ with

$$
G_{M_{1}}\left(\widetilde{I}_{2} \cap \widetilde{I}_{p}\right)=G_{M_{2}}\left(I_{2}\right) G_{p}\left(I_{p}\right),
$$

and that all subsets in the image of $f_{M_{1}}$ are obtained in this way.

This construction allows us to build recursively the image of $f_{M}$. It also enables us to find how many classes have the same image. Therefore, one can compute $G_{M}(I)$ without knowing $g_{M}(I)$.

Let us give an example. For

$$
M=5592405=3 \cdot 5 \cdot 7 \cdot 13 \cdot 17 \cdot 241=\left(2^{24}-1\right) / 3,
$$

we have $\omega=24$. There are 16401 subsets in the image of $f_{M}$, which is much fewer than $2^{24}$. Each of these subsets is obtained in $r$ ways, with $1 \leq r \leq$ 250068. Only subsets of cardinality at most 3 lead to an improved upper bound. The empty set appears 48 times. Each of the singletons from $\mathbb{Z} / 24 \mathbb{Z}$ appears 540 times. For 2 -subsets, the situation is slightly more complicated to describe. The subsets of the form $\{a, a \pm 8\}$ appear 3625 times (there are 24 of them) while those of the form $\{a, a+12\}$ appear 7170 times (there are 12 of them). There are 224 interesting 3 -subsets, appearing 3, 6, 225 or 9520 times.

This method requires much less memory than the algorithm from the previous subsection. It is still possible to save a bit more memory. Indeed, the representation problem (by an invertible plus a power of 2 ) is invariant when multiplied by a power of 2 . So we can use a representative of a collection of subsets, each of them being obtained by translation from the representative, instead of subsets of $\mathbb{Z} / \omega \mathbb{Z}$.

The best result found so far is given by

$$
M=3 \cdot 5 \cdot 7 \cdot 11 \cdot 13 \cdot 17 \cdot 19 \cdot 31 \cdot 41 \cdot 73 \cdot 241 \cdot 257 .
$$

It leads to the improvement

$$
\bar{d}<0.9818818607968211912960156368,
$$

and the upper bound from Theorem 1 follows. This computation took 35 minutes on an Intel Xeon $2.4 \mathrm{GHz}$ with a memory stack of $2.1 \mathrm{~GB}$. Indeed, the real limitation is the memory. Note that during the computations, subsets for which $G_{M}(I)$ was quite large and thus unlikely to contribute to the density were dropped (still there were a total of 4469837 different subsets at the end). Hence the density obtained may be a little greater than the actual density for this value of $M$.

Addendum. The referee informed the authors that, while the paper was being refereed, János Pintz improved on the lower bound. In a paper to 
appear in Acta Math. Hungar., he showed $\underline{d} \geq 0.18734$ by a more elaborate method.

\section{References}

[1] P. Erdős, On integers of the form $2^{k}+p$ and some related problems, Summa Brasil. Math. 2 (1950), 113-123.

[2] D. R. Heath-Brown and J.-C. Puchta, Integers represented as a sum of primes and powers of two, Asian J. Math. 6 (2002), 535-565.

[3] J. Pintz and I. Z. Ruzsa, On Linnik's approximation to Goldbach's problem, I, Acta Arith. 109 (2003), 169-194.

[4] F. Romani, Computations concerning primes and powers of two, Calcolo 20 (1983), 319-336.

[5] N. P. Romanov, Über einige Sätze der additiven Zahlentheorie, Math. Ann. 109 (1934), 668-678.

Institut Camille Jordan

CNRS UMR 5208 Mathématiques

Université Claude Bernard Lyon 1

43 boulevard du 11 novembre 1918

69622 Villeurbanne Cedex, France

E-mail: Laurent.Habsieger@math.univ-lyon1.fr roblot@math.univ-lyon1.fr

Received on 8.2.2005

and in revised form on 20.12.2005 